\title{
Presence of presynaptic neurotoxin complexes in the venoms of Australo-Papuan death adders (Acanthophis spp.)
}

\author{
Benjamin Blacklow ${ }^{a}$, Nicki Konstantakopoulos ${ }^{b}$, Wayne C. Hodgson ${ }^{b}$, \\ Graham M. Nicholson ${ }^{a,{ }^{*}}$ \\ ${ }^{a}$ Neurotoxin Research Group, Department of Medical \& Molecular Biosciences, \\ University of Technology, Sydney, NSW, Australia \\ ${ }^{\mathrm{b}}$ Monash Venom Group, Department of Pharmacology, Monash University, Victoria, \\ Australia
}

\# Ethical statement: The authors declare that all animal experiments described in the paper comply with Australian animal ethics regulations.

* Corresponding author. Associate Professor Graham Nicholson, PhD Neurotoxin Research Group

Department of Medical \& Molecular Biosciences

University of Technology, Sydney

PO Box 123

Broadway, NSW, 2007

Australia

Tel.: +61-2-9514-2230; fax: +61-2-9514-8206

E-mail address: Graham.Nicholson@uts.edu.au (G. Nicholson). 


\begin{abstract}
Australo-papuan death adders (Acanthophis spp.) are a cause of serious envenomations in Papua New Guinea and northern Australia often resulting in neurotoxic paralysis. Furthermore, victims occasionally present with delayed-onset neurotoxicity that sometimes responds poorly to antivenom or anticholinesterase treatment. This clinical outcome could be explained by the presence of potent snake presynaptic phospholipase $A_{2}$ neurotoxin (SPAN) complexes and monomers, in addition to long- and short-chain postsynaptic a-neurotoxins, that bind irreversibly, block neurotransmitter release and result in degeneration of the nerve terminal. The present study therefore aimed to determine within-genus variations in expression of high molecular mass SPAN complexes in the venoms of six major species of Acanthophis, four geographic variants of $A$. antarcticus. Venoms were separated by size-exclusion liquid chromatography under non-denaturing conditions and fractions corresponding to proteins in the range of 22 to $>60 \mathrm{kDa}$ were subjected to pharmacological characterisation using the isolated chick biventer cervicis nerve-muscle (CBCNM) preparation. All venoms, except $A$. wellsi and $A$. pyrrhus, contained high mass fractions with phospholipase $A_{2}$ activity that inhibited twitch contractions of the CBCNM preparation. This inhibition was of slow onset, and responses to exogenous nicotinic agonists were not blocked, consistent with the presence of SPAN complexes. The results of the present study indicate that clinicians may need to be aware of possible prejunctional neurotoxicity following envenomations from $A$. antarcticus (all geographic variants except perhaps South Australia), A. praelongus, A. rugosus and A. laevis species, and that early antivenom intervention is important in preventing further development of toxicity.
\end{abstract}

Keywords: Presynaptic snake neurotoxin; SPAN; death adder; Acanthophis spp.; neurotransmitter release; phospholipase $A_{2}$ 


\section{Introduction}

The family Elapidae consists of a variety of highly venomous snake genera, including the death adders (Acanthophis spp.), which are considered elapids despite their viperlike appearance and behaviour. In addition, death adders have semi-mobile fangs which results in them being commonly regarded as the most evolved of all elapids and morphologically transitional between the Elapidae and Viperidae. Indeed, death adders are the widest ranging of the Australian elapids with a variety of species found throughout continental Australia, Irian Jaya, Papua New Guinea (PNG) and some eastern Indonesian islands including the Maluku islands of Seram (formerly Ceram), Halmahera, Obi and Tanimbar (Lalloo et al., 1996; Wüster et al., 2005). This wide range and ecological specialization of Acanthophis is consistent with its relatively early divergence from its closest sister lineage around 9.1 Mya (Sanders et al., 2008).

Acanthophis species are the second most common cause of envenomation in the Central Province and National Capital District of PNG, second only to the Papuan taipan (Oxyuranus scutellatus canni). They have been reported to be responsible for ca. $11 \%$ of patients admitted for envenomation in these areas between January 1990 and June 1992 (Lalloo et al., 1994). The death adder has additionally been reported to be the cause of most serious envenomations in the Madang region of PNG (Hudson and Pomat, 1988) while in northern Australia death adder envenomation is also considered a serious problem (Currie, 2003). General symptoms of envenomation from Acanthophis species can include pain in regional lymph nodes, and non-specific systemic features such as abdominal pain, headache, drowsiness, nausea and vomiting. More problematic are neurological symptoms that typically involve ptosis, dysarthria, dysphagia, diplopia and flaccid muscle paralysis with death resulting from respiratory failure (Campbell, 1966; Lalloo et al., 1996). Myotoxicity is not usually associated with envenomation but there are clinical reports in PNG of rhabdomyolysis with $A$. rugosus (Lalloo et al., 1996). Significantly, delayed-onset neurotoxicity is a problem in death adder envenomations, occasionally with late presentation of neurotoxicity as the first feature of envenoming (Currie, 1989; Currie, 2003; Lalloo et al., 1996). This has resulted in a recommended hospital admission period of 24 hours for 
patients in PNG and both central and northern Australia, and for children in any region (Currie, 2006).

The primary treatment for death adder envenomation is CSL monovalent death adder antivenom, which has been raised against $A$. antarcticus (White, 1998; White, 2001). Death adder antivenom appears to be quite effective in preventing the progression of neurotoxicity from all species of death adder in Australia and PNG (Campbell, 1966; Lalloo et al., 1996), showing a rapid reversal of paralysis. However, conflicting reports with Australian death adder envenomation have suggested that the neurotoxic effects can be poorly reversed by antivenom or anticholinesterase (neostigmine) if the patient presents late after envenomation (Gunja et al., 2007). In support, an in vitro study involving $A$. antarcticus, $A$. praelongus and $A$. pyrrhus venoms found that all three venoms produced rapid postsynaptic neurotoxicity but antivenom displayed varying efficacy to reverse toxicity following neuromuscular blockade over a 4 hour period. Indeed, there was only a $22 \%$ recovery of contractile responses following complete neuromuscular blockade with $A$. antarcticus, the venom to which the antivenom is raised (Wickramaratna and Hodgson, 2001).

It is commonly regarded that death adder neurotoxicity is mainly postsynaptic in origin, and therefore this suggests that the postsynaptic neurotoxins (so-called ' $\alpha$-' or 'threefingered' neurotoxins) present within death adder venoms may not be completely reversible or are 'pseudo-irreversible'. However, rapidly developing neurotoxicity from postsynaptic neurotoxins conceals the action of any underlying presynaptic neurotoxins or myotoxins that may be present in the venom, which typically have slower onsets of activity but bind irreversibly (Kelly and Brown, 1974; Wilson et al., 1995). In the case of patients with delayed-onset or slowly-developing neurotoxicity, presynaptic neurotoxins or myotoxins may play a significant role in the speed of recovery following antivenom therapy due to the irreversible nature of their actions, and may go some way to explain the above resistance to antivenom therapy. The presence of myotoxins in the venom of A. praelongus, A. rugosus, A. sp. Seram, and A. wellsi species has been previously determined (Hart et al., 2005; Wickramaratna et al., 2003a; Wickramaratna et al., 2003b) and rhabdomyolysis has been reported clinically with $A$. rugosus envenomation 
(Lalloo et al., 1996). However, this does not exclude the presence of presynaptic neurotoxins in these venoms, or in other major species or geographic variants of $A$. antarcticus.

It is therefore possible that Acanthophis venoms contain snake presynaptic phospholipase $A_{2}\left(P L A_{2}\right)$ neurotoxins (so-called ' $\beta$-neurotoxins' or SPANs) that bind irreversibly to motor nerve terminals (Rossetto and Montecucco, 2008). In support, highly toxic heteromultimeric SPANs have been identified and pharmacologically characterized from the venom of a number of other Australo-Papuan elapid snake venoms, including, paradoxin (inland taipan, Oxyuranus microlepidotus; (Fohlman, 1979; Hodgson et al., 2007), taipoxin (coastal taipan, O. scutellatus; (Fohlman et al., 1976), cannitoxin (Papuan taipan, O. scutellatus canni; (Kuruppu et al., 2005b) and textilotoxin (common brown snake, Pseudonaja textilis; (Su et al., 1983; Wilson et al., 1995) and the monomeric notexin (tiger snake, Notechis s. scutatus; (Cull-Candy et al., 1976). These are potent presynaptically-active neurotoxins with an $L D_{50}$ of $1-25 \mu \mathrm{g} / \mathrm{kg}$ (mouse i.v. or i.p.). Furthermore, a protein from $A$. antarcticus venom with significant Nterminal sequence homology to the $y$-chain of the heterotrimeric SPAN taipoxin has been previously reported (Fry et al., 2002), suggestive of the presence of a SPAN in Acanthophis venom.

SPANs can cause irreversible neurotoxicity via the depletion of neurotransmitter, due to a modest increase in transmitter release and a block of vesicle recycling, as well as the degeneration of motor nerve terminals and intramuscular axons (Harris et al., 2000). Regeneration and functional reinnervation can take several days (Dixon and Harris, 1999; Harris et al., 2000) and in humans paralysis resulting from SPANs not promptly treated with antivenom can last many days. Some patients even require artificial ventilation and, in isolated cases, intensive care for prolonged periods (Connolly et al., 1995; Pearn, 1971; Trevett et al., 1995). While a number of monomeric PLA 2 proteins have been isolated from death adder venom (Chow et al., 1998; Sim, 1998; van der Weyden et al., 2000; van der Weyden et al., 1997), none of these have been characterized pharmacologically and there have be no previous reports of attempts at isolating multimeric SPAN complexes in the venom of Acanthophis spp. 
Given the high potency of SPAN complexes from other Australian elapid venoms, the aim of this study was to determine whether the venoms of various species and geographic variants of Australian death adders also contain SPAN complexes. The presence of such components could explain reports of poorly reversible neurotoxicity in post-envenomated patients not treated promptly with antivenom.

\section{Materials and methods}

\subsection{Venom source and storage}

Lyophilized pooled Acanthophis spp. venoms were provided by Venom Supplies (Tanunda, South Australia). The death adders were collected from their natural habitats in Australia and PNG. A degree of controversy exists regarding the taxonomy of the death adders (Williams et al., 2006; Wüster et al., 2005; Wüster et al., 1999), with as many as 15 separate species reported by various sources. Therefore, reporting the locations of Acanthophis snakes used in this study is crucial to allow any subsequent interpretation of these results in the light of any future revision of the taxonomy of Acanthophis. Presently the common death adder, A. antarcticus, is the widest ranging species found in the coastal areas of Australia with two disjoint geographic ranges, New South Wales (NSW)/Queensland (QLD) and South Australia (SA)/Western Australia (WA). Venom was obtained from populations in Sydney (A. antarcticus NSW), Mt Glorious northwest of Brisbane (A. antarcticus QLD), Middleback Ranges close to Whyalla (A. antarcticus SA), and Darling Range ( $A$. antarcticus WA). Other Acanthophis spp. included in this study are the northern death adder $A$. praelongus (sourced from Cairns, QLD), desert death adder A. pyrrhus (north of Alice Springs, Northern Territory), as well as the Pilbara death adder $A$. wellsi (Pilbarra, WA). The rough-scaled $A$. rugosus (sourced from Merauke, West Papua, Indonesia) and smooth-scaled A. laevis (Seram, Indonesia) death adders are found in PNG, with $A$. laevis also being found on the surrounding Indonesian islands. Although, in the past, the venom samples collected from Seram specimens have been referred to as $A$. sp. Seram, it has been reported that A. sp. Seram belongs to the $A$. laevis complex (Wüster et al., 2005). This study will 
therefore refer to $A$. sp. Seram as $A$. laevis. For each geographic variant or major species, venoms were collected and pooled to minimize the effects of individual variations (Chippaux et al., 1991). Venoms were collected from fang tips through the specimen biting down on a parafilm covered specimen bottle and injecting the venom into a polyethylene container, in combination with the use of polyethylene pipette tip to milk any residual venom from the fang. The venoms were then lyophilised and stored at $-20^{\circ} \mathrm{C}$ until required.

\subsection{Size-exclusion FPLC under non-denaturing conditions}

Bioassay-guided isolation of toxins from whole venom was performed using a Shimadzu CLASS-VP HPLC system. Prior to separation, samples were suspended in equilibration buffer and centrifuged at $10,000 \mathrm{rpm}$ for $5 \mathrm{~min}$, with the resulting supernatant (equivalent to ca. $3 \mathrm{mg}$ of venom) applied to a Superdex G-75 column (10 x $300 \mathrm{~mm}, 13$ $\mu \mathrm{m}$; GE Healthcare, Sydney, NSW, Australia) equilibrated with ammonium acetate (0.1 $\mathrm{M}, \mathrm{pH}$ 6.8). Fractions were eluted using an isocratic gradient at a flow rate of 0.75 $\mathrm{ml} / \mathrm{min}$. Elution profiles were monitored at 280 and $214 \mathrm{~nm}$, however only the profile obtained at $280 \mathrm{~nm}$ is shown. Resulting fractions were screened for both neurotoxic and myotoxic activity using the chick biventer cervicis nerve-muscle (CBCNM) preparation (see section 2.4) and phospholipase $A_{2}\left(P L A_{2}\right)$ activity (see section 2.5). The approximate molecular mass of any SPAN complex was determined by gel filtration under non-reducing conditions on the Superdex G-75 column equilibrated with ammonium acetate buffer $(0.1 \mathrm{M} ; \mathrm{pH} 6.8)$. The column was calibrated with the series of known standards $(6.5-66 \mathrm{kDa})$ using the molecular mass marker kit for gel filtration chromatography (lot no. 093K9307; MWGF-70; Sigma-Aldrich, St. Louis, MO) at a flow rate of $0.75 \mathrm{ml} / \mathrm{min}$. The void volume $\left(\mathrm{V}_{0}\right)$ of the column was determined by running blue dextran $(2,000 \mathrm{kDa})$, and the elution volume $\left(\mathrm{V}_{\mathrm{e}}\right)$ was calculated for each molecular mass marker. The molecular mass range of SPAN complexes were then determined from interpolation of a plot of log molecular mass versus retention ratio $\left(\mathrm{V}_{\mathrm{e}} / \mathrm{V}_{\mathrm{o}}\right)$ of the standards. 


\subsection{Bicinchoninic Acid Protein (BCA) Assay:}

Following lyophilisation, the protein concentrations of purified high molecular mass fractions were determined using the Quantipro ${ }^{\mathrm{TM}}$ BCA assay kit (Sigma-Aldrich). Protein contents between $5-200 \mu \mathrm{g} / \mathrm{ml}$ were detected at $560 \mathrm{~nm}$ by a Titertek Multiscan Plus MKII (type 313) plate reader (Flow Laboratories Australasia, North Ryde, NSW). A standard curve was created using bovine serum albumin from which protein concentrations of the venom fractions were interpolated. Yields of the high molecular mass fraction (22 to $>66 \mathrm{kDa}$ ) were then expressed as a percentage of whole venom loaded onto the column ( $3 \mathrm{mg})$.

\subsection{Isolated chick biventer cervicis nerve-muscle preparation}

Isolated fractions were tested for neurotoxic and myotoxic activity using the isolated CBCNM preparation (Ginsborg and Warriner, 1960). Male Australorps chicks aged 1-7 days were euthanised with $\mathrm{CO}_{2}$ and exsanguinated. The biventer cervicis muscle with attached nerve was dissected and placed in an organ bath (5 or $8 \mathrm{ml})$ under $1 \mathrm{gram}$ of resting tension. The organ bath contained Krebs-Henseleit solution of the following composition (in $\mathrm{mM}$ ): $\mathrm{NaCl}, 118.4 ; \mathrm{KCl}, 4.7 ; \mathrm{MgSO}_{4}, 1.2 ; \mathrm{KH}_{2} \mathrm{PO}_{4}, 1.2 ; \mathrm{NaHCO}_{3}, 25.0$; D-glucose, 11.1; $\mathrm{CaCl}_{2}$, 2.5, which was bubbled with carbogen $\left(95 \% \mathrm{O}_{2}\right.$ and $\left.5 \% \mathrm{CO}_{2}\right)$ and maintained at $34^{\circ} \mathrm{C}$. Indirect stimulation was applied to the motor nerve using supramaximal $30 \mathrm{~V}$ square-wave pulses of $0.2 \mathrm{~ms}$ duration at $0.1 \mathrm{~Hz}$ with a Grass S88 stimulator. Contractions were measured using an isometric force transducer (ADInstruments, Belle Vista, NSW, Australia). The nerve-muscle preparation was allowed to equilibrate for 30 minutes. Exclusive electrical stimulation of the nerve was confirmed by complete blockade of twitches using $10 \mu \mathrm{M} d$-tubocurarine. Washout was then repeated until twitch tension returned to its original amplitude. Contractures to various exogenous agonists were subsequently recorded in the absence of electrical stimulation before, and after, incubation with the SPAN. The final bath concentrations and periods of incubation for each exogenous agonist were as follows: acetylcholine $(\mathrm{ACh}, 1 \mathrm{mM})$ for $30 \mathrm{~s}$; carbachol $(\mathrm{CCh}, 20 \mu \mathrm{M})$ for $60 \mathrm{~s}$; potassium chloride $(\mathrm{KCl}, 40$ 
$\mathrm{mM}$ ) for $30 \mathrm{~s}$ (Harvey et al., 1994). After each agonist incubation period, washout was repeated until a stable baseline was observed. Following addition of SPAN $(10 \mu \mathrm{g} / \mathrm{ml})$, twitch tension amplitude was monitored for 4 hours, or until twitches were abolished. Time-matched controls confirmed that muscle fatigue was not significant up to 4 hours after introduction of muscles into the organ bath. Muscle tension from isometric force transducers was amplified using a ML221 bridge amplifier (ADInstruments) and recorded using a Powerlab 2/25 system (ADInstruments) connected to an iMac or PC computer. Data was digitised at $140 \mathrm{~Hz}$ and filtered using 50/60 Hz high-pass and 20$200 \mathrm{~Hz}$ low-pass filters. Muscle tension was recorded using Chart v5.5.4 software (ADInstruments).

\subsection{Phospholipase $A_{2}\left(P L A_{2}\right)$ activity assay}

The PLA ${ }_{2}$ activity of whole venoms and isolated toxins were determined using a colorimetric $\mathrm{SPLA}_{2}$ assay kit (Cayman Chemical Ltd, Ann Arbor, MI, USA). The presence of a $\mathrm{PLA}_{2}$ enzyme catalyses the hydrolysis of the thio ester bond on the phospholipid substrate (1,2-dithio analog of diheptanoyl phosphohatidylcholine) at the sn-2 position. This results in the production of free thiols which are detected by 5,5'dithio-bis-(2-nitrobenzoic acid). Colour development was monitored using a BIO-RAD model 3550-UV microplate reader at $405 \mathrm{~nm}$. Measurements were made every $20 \mathrm{~s}$ for $5 \mathrm{~min}$. PLA 2 activity was calculated as micromoles of substrate hydrolysed per minute per milligram of enzyme $(\mu \mathrm{mol} / \mathrm{min} / \mathrm{mg})$, with bee (Apis mellifera) venom as a positive control.

\subsection{Mass spectrometry}

MALDI-TOF mass spectrometry was performed on a Voyager DE-Pro instrument (Applied Biosystems, Foster City, CA, USA) operated in positive linear mode. Crude venom samples were dissolved in $10 \mathrm{ml} \mathrm{HPLC-grade} \mathrm{water} \mathrm{and} 0.5 \mathrm{ml}$ samples were mixed on-plate in a 1:2 ratio with 3,5 dimethoxy-4-hydroxycinnamic acid (sinapinic acid) 
matrix made up in acetonitrile/water/trifluoroacetic acid (30:70:0.1). Spectra were externally calibrated with a mixture of synthetic proteins $(5,700-17,000$ Da mass range, LaserBio Labs, France) using the same matrix system. Spectra were processed using the Data Explorer software package (Applied Biosystems).

\subsection{Chemicals and drugs}

Unless otherwise stated, all chemicals and drugs were purchased from Sigma-Aldrich, Castle Hill, NSW, Australia.

\subsection{Data analysis}

Twitch contraction amplitude was expressed as a percentage of initial amplitude, prior to addition of the SPAN. Contractile responses to exogenous agonists were expressed as a percentage of their amplitude prior to addition of the SPAN. To compare the neurotoxicity of venoms, the time taken to cause $90 \%$ inhibition of nerve-mediated twitch contractions $\left(t_{90}\right)$ was determined as a quantitative measure of potency. Values for $t_{90}$ were calculated for each experiment by determining the elapsed time, after venom addition, to $10 \%$ of the initial twitch amplitude, and then the means and standard error of the means were calculated. Where indicated, statistical significance was determined by one-way analysis of variance (ANOVA) or paired Student's t-test. All ANOVAs were followed by a Bonferroni-corrected multiple $t$-test. Statistical analyses were performed using the PRISM 4.0 software package (GraphPad Software, San Diego, CA, USA). A $p$-value of 0.05 was employed for all statistical analyses.

\section{Results}

\subsection{Venom fractionation}

Separation of Acanthophis spp. whole venoms by size-exclusion FPLC under nonreducing conditions produced a characteristic pattern of fractions from all death adder 
venom samples (Fig. 1). In general, resultant chromatograms displayed 4-6 major peaks. Nevertheless, variation in chromatographic profiles was evident between the regional variants of $A$. antarcticus as well as other Acanthophis spp., where the variation was more pronounced. Given that SPAN complexes have molecular masss ranging between $21 \mathrm{kDa}$ (the covalently-linked heterodimer $\beta$-bungarotoxin from Bungarus fasciatus; (Chu et al., 1995) and ca. $87 \mathrm{kDa}$ (the heterohexameric textilotoxin; (Aquilina, 2009) fractions falling into this molecular mass range were collected for neurotoxicity testing. Following calibration of the size-exclusion column using a set of molecular mass markers, the retention times of early-eluting fractions were found to be consistent with a molecular mass range between $22 \mathrm{kDa}$ to $>66 \mathrm{kDa}$ (shaded fractions in Fig. 1Aa and B). Given that the calibration of the size-exclusion Sephadex G-75 column may not be linear beyond $66 \mathrm{kDa}$ (bovine serum albumin was the highest mass standard used), we have elected not to extrapolate beyond this upper limit. Importantly, separation of $A$. wellsi and $A$. pyrrhus venoms did not result in any early-eluting components (Fig. 1Ab) and therefore these venoms were not investigated any further in this study. For the remaining venoms, the yields of this high molecular mass fraction, as determined by the BCA assay, appear to vary significantly, with $A$. laevis possessing the highest yield of $14.9 \% \mathrm{w} / \mathrm{w}$ while $A$. antarcticus $(\mathrm{SA})$ had the lowest yield of $2.7 \% \mathrm{w} / \mathrm{w}$. The percent yields of the prejunctional neurotoxin component from each Acanthophis venom are shown in Table 1.

\subsection{Neurotoxicity studies}

Apart from $A$. antarcticus (SA) venom, all high molecular mass fractions produced timedependant inhibition of nerve-evoked twitch contractions within 240 min ( $n=3-4$; Fig. $2 \mathrm{~A}$ ), whereas with time-matched controls there was no significant inhibition of twitch tension over the 240 min period (Fig. 2B). The mean $t_{90}$ values ranged from 59 to 85 min (see Table 1). There was no significant difference between high molecular mass fractions from all venoms except that from $A$. antarcticus (SA) venom, which failed to reach $90 \%$ block after $240 \mathrm{~min}$. Because experiments were terminated after $240 \mathrm{~min}$, the $t_{90}$ value for the high molecular mass fraction from $A$. antarcticus (SA) venom was 
not determined. A triphasic timecourse of action characterized by depressionenhancement-blockade of neurotransmission was evident with the high molecular mass fraction from the WA and NSW geographic variants of $A$. antarcticus as has been previously reported for a variety of other SPANs in mammalian nerve-muscle preparations (Su and Chang, 1984; Wilson et al., 1995). The remaining Acanthophis spp. venom fractions did not overtly display this triphasic timecourse, consistent with that often seen for SPANs in avian neuromuscular preparations (Beghini et al., 2004; Kuruppu et al., 2005b).

Importantly, following complete neuromuscular blockade by all high molecular mass fractions, responses to cholinergic agonists were still present whereas the negative control had no significant inhibitory effect on the contractures produced by exogenous agonists ( $n=3-4$; Fig. 3). While $A$. laevis caused the only statistically significant inhibition of ACh responses (one-way ANOVA, $p<0.01, n=3$ ), contractures were not completely abolished. Moreover, the responses to carbachol were not significantly inhibited by any high molecular mass fraction (one-way ANOVA, $p>0.05, n=3-4$ ). Interestingly, the responses to carbachol were enhanced in controls and venom fractions but this has been previously reported in this preparation (Kuruppu et al., 2005a; Kuruppu et al., 2005b; Lumsden et al., 2005) and may reflect time-dependent sensitization of the tissue to carbachol. All fractions failed to significantly inhibit responses to potassium chloride (one-way ANOVA, $p>0.05, n=3-4$ ) and did not cause a significant change in the baseline tension of the biventer cervicis muscle (data not shown). These actions are consistent with the presence of SPAN complexes in the high molecular mass fraction that inhibit neurotransmitter release.

\section{3. $P L A_{2}$ activity}

The PLA 2 activity of whole venoms and isolated prejunctional neurotoxic components showed some variability (Fig. 3). Whole venoms and isolated components all possessed $\mathrm{PLA}_{2}$ activity, however the individual activities were much lower than the positive control (bee venom), which had a PLA 2 activity of $181.8 \pm 9.7 \mu \mathrm{mol} / \mathrm{min} / \mathrm{mg}$. PLA $A_{2}$ activity of the 
weakly-active $A$. antarcticus (SA) component possessed the lowest enzymatic activity, however it was comparable to the A. laevis component, which possessed marked neurotoxicity.

\subsection{Mass spectrometry}

Given that there was considerable variation in the neurotoxicity of the regional variants of $A$. antarcticus, the mass profile of NSW, QLD and SA geographic variants of $A$. antarcticus venoms were examined. The MALDI-TOF mass spectra of these venoms were dominated by high-intensity ions at ca. 6400-6700, 6700-7100, 7200-8900 and $12800-14000 \mathrm{~m} / \mathrm{z}$ ranges (Fig. 5). This is consistent with the presence of Kunitz-type protease inhibitors, short-chain a-neurotoxins, long-chain a-neurotoxins and monomeric $\mathrm{PLA}_{2}$ neurotoxins, respectively. Allowing for minor measurement differences resulting from external calibration, it appears that these venoms contain a number of equivalent $\mathrm{PLA}_{2}$ components particular at ca. $\mathrm{m} / \mathrm{z}$ 12847, 12901, 13461, 13816, and 13916, although the molecular identity of these proteins would need to be confirmed via Edman sequencing. Two of these masses are similar to those previously determined for the monomeric PLA 2 proteins acanthoxin A1 (12842 Da) and acanthoxin A2 (12896 Da) (van der Weyden et al., 1997) as well as the platelet aggregation inhibitors acanthin I (12845 Da) and acanthin II (12,896 Da) (Chow et al., 1998) from A. antarcticus venom (unnamed variants). Furthermore, the venoms also contained a number of conserved masses ca. $\mathrm{m} / \mathrm{z}$ 6711, 6752, 6860, consistent with short-chain a-neurotoxins and $\mathrm{m} / \mathrm{z}$ 8185 and 8856, consistent with long-chain a-neurotoxins (see highlighted masses in Fig. 5). An additional mass at $m / z 6653$, present in all three venoms, is consistent with a Kunitz-type protease inhibitor previously identified in $A$. antarcticus venoms (Fry et al., 2002).

\section{Discussion}


Excluding the venoms of $A$. wellsi and $A$. pyrrhus, all species including the geographic variants of death adder venoms contained early eluting fractions with masses estimated between 22 and $>66 \mathrm{kDa}$. Apart from $A$. antarcticus (SA), each of these high molecular mass fractions caused a block of muscle contractility consistent with strong prejunctional neurotoxicity. Each of these components also possessed significant PLA 2 enzymatic activity. Given that these components represent a significant portion of the whole venom, they would therefore contribute to the overall toxicity of the venom. The estimated size, neurotoxicity and $\mathrm{PLA}_{2}$ enzymatic activity indicates that these isolated venom components most likely contain SPANs that are likely composed of a multimeric structure. It is interesting to speculate therefore that these SPANs have a similar heterotrimeric complex of ca. $45 \mathrm{kDa}$ much like previously isolated SPANs such as taipoxin, cannitoxin and paradoxin from various species of Oxyuranus (Fohlman, 1979; Fohlman et al., 1976; Kuruppu et al., 2005b). A number of components with molecular masses comparable to other snake monomeric $\mathrm{PLA}_{2}$ neurotoxins and inhibitors of platelet aggregation (ca. 12.8-14 kDa) were conserved in venoms from NSW, QLD and SA geographic variants of $A$. antarcticus. It is therefore surprising that $A$. antarcticus (SA) venom, which possessed only a weakly active high molecular mass fraction, contained all five of these proteins. This suggests that other components in this mass range form the subunits of the SPAN complex. While there exists the possibility that this fraction could contain blockers of voltage-gated sodium or calcium channels to inhibit neurotransmission, the high mass and PLA $A_{2}$ activity consistent with other SPANs would argue against this. Further biochemical studies of several of these venoms is presently being undertaken to ascertain the subunit structure and confirm the pharmacological basis for this prejunctional neurotoxicity (unpublished data).

Of note is the absence of a high molecular mass SPAN complex in the venoms of $A$. wellsi and $A$. pyrrhus. These two well-differentiated species are believed to belong to a separate clade distinct from other species of Acanthophis (Wüster et al., 2005), supporting their unique size-exclusion chromatographic profile. Importantly, these two venoms appear to have a higher yield of monomeric PLA $\mathrm{A}_{2}$ neurotoxins (ca. 12.8-14 $\mathrm{kDa}$ ) and/or postsynaptic a-neurotoxins (ca. 6.7-8.9 kDa) based on the molecular mass of the large eluting peak between 17 and $22 \mathrm{~min}$ (Fig. 1Ab). 
Notably there was an apparent lack of any overt myotoxicity in the high molecular mass fraction of all venoms, as evidenced by a lack of inhibition of $\mathrm{KCl}$ responses and the absence of any change in baseline tension in the CBCNM preparation. This would suggest that previously identified myotoxins from A. rugosus (Wickramaratna et al., 2003a) and A. sp. Seram (A. laevis in this study; (Hart et al., 2005) appear to exist as monomeric $\mathrm{PLA}_{2}$ myotoxins (13082-13896 Da), eluting later in the size-exclusion chromatogram rather than as a complex of higher molecular mass. Thus none of the high molecular mass SPAN complexes in Acanthophis venoms appear to possess significant myotoxic activity, as has been described for the $45 \mathrm{kDa}$ heterotrimeric SPAN, taipoxin (Harris et al., 2000; Harris and Maltin, 1982).

The poor reversibility of Acanthophis neurotoxicity particularly $A$. antarcticus, both in vitro (Wickramaratna and Hodgson, 2001) and clinically (Gunja et al., 2007), could be the result of irreversible binding of long-chain snake a-neurotoxins. In support, longchain snake a-neurotoxins such as Aa b (8125 Da, (Kim and Tamiya, 1981), Aa e (8751 Da, (Tyler et al., 1997), acanthophin d (8377 Da, (Sheumack et al., 1990), and aelapitoxin-Aa2a (8850 Da, Blacklow B and Nicholson GM, unpublished observations) have been identified in the venom of various geographic variants of $A$. antarcticus. They appear to bind with high affinity and are poorly reversible (Sheumack et al., 1990); Blacklow B and Nicholson GM, unpublished observations). In the present study, several polypeptides in a mass range consistent with the presence of long-chain a-neurotoxins (ca. 7.2-8.9 kDa) were also confirmed in the venoms of NSW, QLD and SA geographic variants. It is important to note, however, that the toxicity and reversibility of all Acanthophis long-chain a-neurotoxins, except acanthophin $d$ and $\alpha$-elapitoxin-Aa2a, is assumed based on size of toxin and symptoms arising from parenteral administration in mice. Furthermore, none of masses of the previously reported long-chain $\alpha$-neurotoxins were identified in the MALDI-TOF mass profiles in Figure 5. In summary, the present study has identified the presence of SPANs in at least six, of the nine, venoms investigated. If long-chain snake $\alpha$-neurotoxins were also present in any of these other venoms they should only serve to increase the poor reversibility of neurotoxicity seen with inadequate use of antivenom. 
Reduction in the ACh response by the high molecular mass fraction of $A$. laevis venom could be due to the presence of postsynaptic PLA ${ }_{2}$ complexes such as has been described for vipoxin a $27.5 \mathrm{kDa}$ heterodimeric $\mathrm{PLA}_{2}$ complex from the Bulgarian viper (Vipera ammodytes meridionalis) (Banumathi et al., 2001). On the hand, the reduction in ACh response could also be due to the presence of an acetylcholinesterase in the high molecular mass fraction in amounts sufficient to hydrolyse exogenous acetylcholine. Acetylcholinesterase has been characterized previously from Bungarus fasciatus venom (Cousin et al., 1996) and matched to several 70-kDa proteins in the venoms of several Australian elapids, including A. antarcticus, in a recent proteomic study (Birrell et al., 2007). However, neither of these possibilities is likely given that responses to carbachol, a nicotinic agonist resistant to the actions of acetylcholinesterase, were not significantly inhibited at complete neuromuscular blockade.

The effectiveness of prior administration of antivenom $(5 \mathrm{U} / \mathrm{ml})$ to completely neutralize the in vitro neurotoxicity of venom from all major species and geographic variants of Acanthophis has been previously confirmed (Fry et al., 2001). Therefore, considering the present findings that the majority of death adder venoms contain SPANs, early intervention with antivenom is critically important in preventing severe prolonged envenomation. The results of the present study indicate that clinicians may need to be aware of possible prejunctional neurotoxicity following envenomation from $A$. antarcticus (all geographic variants except SA), A. praelongus, A. rugosus, or A. laevis species. Unfortunately, there are no current treatments that prevent the destruction of the nerve terminal once the process of degeneration has been initiated, enhance the repair of the structural damage to the peripheral innervation or increase the rate at which the victims recover neuromuscular function. This would suggest that early antivenom intervention is essential to preventing the further development of toxicity caused by these snakes.

\section{Acknowledgements}


This work was supported in part by an Australian Postgraduate Award to BB. The authors wish to thank Dr Pierre Escoubas, Université de Nice - Sophia Antipolis, IPMC, CNRS, Valbonne, France who carried out the MALDI-TOF mass spectrometry.

\section{References}

Aquilina, J.A., 2009. The major toxin from the Australian Common Brown Snake is a hexamer with unusual gas-phase dissociation properties. Proteins 75, 478-485.

Banumathi, S., Rajashankar, K.R., Nötzel, C., Aleksiev, B., Singh, T.P., Genov, N., Betzel, C., 2001. Structure of the neurotoxic complex vipoxin at $1.4 \AA$ resolution. Acta Crystallogr. D Biol. Crystallogr. 57, 1552-1559.

Beghini, D.G., Rodrigues-Simioni, L., Toyama, M.H., Novello, J.C., da Cruz-Höfling, M.A., Marangoni, S., 2004. Neurotoxic and myotoxic actions of crotoxin-like and Crotalus durissus cascavella whole venom in the chick biventer cervicis preparation. Toxicon 43, 255-261.

Birrell, G.W., Earl, S.T.H., Wallis, T.P., Masci, P.P., de Jersey, J., Gorman, J.J., Lavin, M.F., 2007. The diversity of bioactive proteins in Australian snake venoms. Mol. Cell. Proteomics 6, 973-986.

Campbell, C.H., 1966. The death adder (Acanthophis antarcticus): the effect of the bite and its treatment. Med. J. Aust. 2, 922-925.

Chippaux, J.P., Williams, V., White, J., 1991. Snake venom variability: methods of study, results and interpretation. Toxicon 29, 1279-1303.

Chow, G., Subburaju, S., Kini, R.M., 1998. Purification, characterization, and amino acid sequence determination of acanthins, potent inhibitors of platelet aggregation from Acanthophis antarcticus (common death adder) venom. Arch. Biochem. Biophys. 354, 232-238.

Chu, C.C., Li, S.H., Chen, Y.H., 1995. Resolution of isotoxins in the $\beta$-bungarotoxin family. J. Chromat. A 694, 492-497.

Connolly, S., Trevett, A.J., Nwokolo, N.C., Lalloo, D.G., Naraqi, S., Mantle, D., Schofield, I.S., Fawcett, P.R., Harris, J.B., Warrell, D.A., 1995. Neuromuscular effects of Papuan Taipan snake venom. Ann. Neurol. 38, 916-920. 
Cousin, X., Bon, S., Duval, N., Massoulié, J., Bon, C., 1996. Cloning and expression of acetylcholinesterase from Bungarus fasciatus venom. A new type of $\mathrm{COOH}$-terminal domain; involvement of a positively charged residue in the peripheral site. J. Biol. Chem. 271, 15099-15108.

Cull-Candy, S.G., Fohlman, J., Gustavsson, D., Lullmann-Rauch, R., Thesleff, S., 1976. The effects of taipoxin and notexin on the function and fine structure of the murine neuromuscular junction. Neuroscience 1, 175-180.

Currie, B.J., 1989. Urban envenomation (Letter). Med. J. Aust. 151, 724.

Currie, B.J., 2003. Snakebite in Australia: moving from anecdotes to prospective studies. Emerg. Med. (Fremantle, WA) 15, 406-408.

Currie, B.J., 2006. Treatment of snakebite in Australia: the current evidence base and questions requiring collaborative multicentre prospective studies. Toxicon 48,941 956.

Dixon, R.W., Harris, J.B., 1999. Nerve terminal damage by $\beta$-bungarotoxin: its clinical significance. Am. J. Pathol. 154, 447-455.

Fohlman, J., 1979. Comparison of two highly toxic Australian snake venoms: the taipan (Oxyuranus s. scutellatus) and the fierce snake (Parademansia microlepidotus). Toxicon 17, 170-172.

Fohlman, J., Eaker, D., Karlsoon, E., Thesleff, S., 1976. Taipoxin, an extremely potent presynaptic neurotoxin from the venom of the Australian snake taipan (Oxyuranus s. scutellatus). Isolation, characterization, quaternary structure and pharmacological properties. Eur. J. Biochem. 68, 457-469.

Fry, B.G., Wickramaratna, J.C., Hodgson, W.C., Alewood, P.F., Kini, R.M., Ho, H., Wüster, W., 2002. Electrospray liquid chromatography/mass spectrometry fingerprinting of Acanthophis (death adder) venoms: taxonomic and toxinological implications. Rapid Commun Mass Spectrom 16, 600-608.

Fry, B.G., Wickramaratna, J.C., Jones, A., Alewood, P.F., Hodgson, W.C., 2001. Species and regional variations in the effectiveness of antivenom against the in vitro neurotoxicity of death adder (Acanthophis) venoms. Toxicol. Appl. Pharmacol. 175, 140-148. 
Ginsborg, B.L., Warriner, J., 1960. The isolated chick biventer cervicis nerve-muscle preparation. Brit. J. Pharmacol. 15, 410-411.

Gunja, N., Ling, M.L., Dowsett, R.P., Murray, L., Isbister, G.K., 2007. Antivenom and neostigmine failure in death adder envenoming. Clin. Toxicol. 45, 389.

Harris, J.B., Grubb, B.D., Maltin, C.A., Dixon, R., 2000. The neurotoxicity of the venom phospholipases $A_{2}$, notexin and taipoxin. Exp. Neurol. 161, 517-526.

Harris, J.B., Maltin, C.A., 1982. Myotoxic activity of the crude venom and the principal neurotoxin, taipoxin, of the Australian taipan, Oxyuranus scutellatus. Br. J. Pharmacol. 76, 61-75.

Hart, A.J., Smith, A.I., Reeve, S., Hodgson, W.C., 2005. Isolation and characterisation of acanmyotoxin-2 and acanmyotoxin-3, myotoxins from the venom of the death adder Acanthophis sp. Seram. Biochem. Pharmacol. 70, 1807-1813.

Harvey, A.L., Barfaraz, A., Thomson, E., Faiz, A., Preston, S., Harris, J.B., 1994. Screening of snake venoms for neurotoxic and myotoxic effects using simple in vitro preparations from rodents and chicks. Toxicon 32, 257-265.

Hodgson, W.C., Dal Belo, C.A., Rowan, E.G., 2007. The neuromuscular activity of paradoxin: a presynaptic neurotoxin from the venom of the inland taipan (Oxyuranus microlepidotus). Neuropharmacology 52, 1229-1236.

Hudson, B.J., Pomat, K., 1988. Ten years of snake bite in Madang Province, Papua New Guinea. Trans. R. Soc. Trop. Med. Hyg. 82, 506-508.

Kelly, R.B., Brown, F.R., 1974. Biochemical and physiological properties of a purified snake venom neurotoxin which acts presynaptically. J. Neurobiol. 5, 135-150.

Kim, H.S., Tamiya, N., 1981. Isolation, properties and amino acid sequence of a longchain neurotoxin, Acanthophis antarcticus b, from the venom of an Australian snake (the common death adder, Acanthophis antarcticus). Biochem. J. 193, 899-906.

Kuruppu, S., Fry, B.G., Hodgson, W.C., 2005a. Presynaptic neuromuscular activity of venom from the brown-headed snake (Furina tristis). Toxicon 45, 383-388.

Kuruppu, S., Reeve, S., Banerjee, Y., Kini, R.M., Smith, A.I., Hodgson, W.C., 2005b. Isolation and pharmacological characterization of cannitoxin, a presynaptic neurotoxin from the venom of the Papuan Taipan (Oxyuranus scutellatus canni). J. Pharmacol. Exp. Ther. 315, 1196-1202. 
Lalloo, D.G., Trevett, A.J., Black, J.A., Mapao, J., Naraqi, S., Owens, D., Hutton, R.A., Theakston, R.D., Warrell, D.A., 1994. Neurotoxicity and haemostatic disturbances in patients envenomed by the Papuan black snake (Pseudechis papuanus). Toxicon 32, 927-936.

Lalloo, D.G., Trevett, A.J., Black, J.A., Mapao, J., Saweri, A., Naraqi, S., Owens, D., Kamiguti, A.S., Hutton, R.A., Theakston, R.D., Warrell, D.A., 1996. Neurotoxicity, anticoagulant activity and evidence of rhabdomyolysis in patients bitten by death adders (Acanthophis sp.) in southern Papua New Guinea. QJM 89, 25-35.

Lumsden, N.G., Fry, B.G., Ventura, S., Kini, R.M., Hodgson, W.C., 2005. Pharmacological characterisation of a neurotoxin from the venom of Boiga dendrophila (mangrove catsnake). Toxicon 45, 329-334.

Pearn, J.H., 1971. Survival after snake-bite with prolonged neurotoxic envenomation. Med. J. Aust. 2, 259-261.

Rossetto, O., Montecucco, C., 2008. Presynaptic neurotoxins with enzymatic activities. Handb. Exp. Pharmacol., 129-170.

Sanders, K.L., Lee, M.S.Y., Leys, R., Foster, R., Keogh, J.S., 2008. Molecular phylogeny and divergence dates for Australasian elapids and sea snakes (hydrophiinae): evidence from seven genes for rapid evolutionary radiations. J. Evol. Biol. 21, 682-695.

Sheumack, D.D., Spence, I., Tyler, M.I., Howden, M.E.H., 1990. The complete amino acid sequence of a post-synaptic neurotoxin isolated from the venom of the Australian death adder snake Acanthophis antarcticus. Comp. Biochem. Physiol. B 95, 45-50.

Sim, K., 1998. Purification and preliminary characterisation of praelongin phospholipases, antiplatelet agents from the snake venom of Acanthophis praelongus. Biochim. Biophys. Acta 1379, 198-206.

Su, M.J., Chang, C.C., 1984. Presynaptic effects of snake venom toxins which have phospholipase $A_{2}$ activity ( $\beta$-bungarotoxin, taipoxin, crotoxin). Toxicon 22, 631-640.

Su, M.J., Coulter, A.R., Sutherland, S.K., Chang, C.C., 1983. The presynaptic neuromuscular blocking effect and phospholipase $A_{2}$ activity of textilotoxin, a potent 
toxin isolated from the venom of the Australian brown snake, Pseudonaja textilis. Toxicon 21, 143-151.

Trevett, A.J., Lalloo, D.G., Nwokolo, N.C., Naraqi, S., Kevau, I.H., Theakston, R.D., Warrell, D.A., 1995. The efficacy of antivenom in the treatment of bites by the Papuan taipan (Oxyuranus scutellatus canni). Trans. R. Soc. Trop. Med. Hyg. 89, 322-325.

Tyler, M.I., Retson-Yip, K.V., Gibson, M.K., Barnett, D., Howe, E., Stöcklin, R., Turnbull, R.K., Kuchel, T., Mirtschin, P., 1997. Isolation and amino acid sequence of a new long-chain neurotoxin with two chromatographic isoforms ( $\mathrm{Aa}$ el and $\mathrm{Ae}$ e2) from the venom of the Australian death adder (Acanthophis antarcticus). Toxicon 35, 555-562.

van der Weyden, L., Hains, P., Broady, K.W., 2000. Characterisation of the biochemical and biological variations from the venom of the death adder species (Acanthophis antarcticus, A. praelongus and A. pyrrhus). Toxicon 38, 1703-1713.

van der Weyden, L., Hains, P., Morris, M., Broady, K.W., 1997. Acanthoxin, a toxic phospholipase $A_{2}$ from the venom of the common death adder (Acanthophis antarcticus). Toxicon 35, 1315-1325.

White, J., 1998. Envenoming and antivenom use in Australia. Toxicon 36, 1483-1492.

White, J., 2001. CSL Antivenom Handbook. CSL Ltd., Melbourne.

Wickramaratna, J.C., Fry, B.G., Aguilar, M.I., Kini, R.M., Hodgson, W.C., 2003a. Isolation and pharmacological characterization of a phospholipase $A_{2}$ myotoxin from the venom of the Irian Jayan death adder (Acanthophis rugosus). Br. J. Pharmacol. 138, 333-342.

Wickramaratna, J.C., Fry, B.G., Hodgson, W.C., 2003b. Species-dependent variations in the in vitro myotoxicity of death adder (Acanthophis) venoms. Toxicol. Sci. 74, 352-360.

Wickramaratna, J.C., Hodgson, W.C., 2001. A pharmacological examination of venoms from three species of death adder (Acanthophis antarcticus, Acanthophis praelongus and Acanthophis pyrrhus). Toxicon 39, 209-216. 
Williams, D., Wuster, W., Fry, B.G., 2006. The good, the bad and the ugly: Australian snake taxonomists and a history of the taxonomy of Australia's venomous snakes. Toxicon.

Wilson, H.I., Nicholson, G.M., Tyler, M.I., Howden, M.E.H., 1995. Induction of giant miniature end-plate potentials during blockade of neuromuscular transmission by textilotoxin. Naunyn-Schmiedeberg's Arch. Pharmacol. 352, 79-87.

Wüster, W., Dumbrell, A., Hay, C., Pook, C., Williams, D., Fry, B., 2005. Snakes across the Strait: trans-Torresian phylogeographic relationships in three genera of Australasian snakes (Serpentes: Elapidae: Acanthophis, Oxyuranus, and Pseudechis). Mol. Phylogen. Evol. 34, 1-14.

Wüster, W., Golay, P., Warrell, D.A., 1999. Synopsis of recent developments in venomous snake systematics, No. 3. Toxicon 37, 1123-1129.

\section{Conflict of interest statements:}

The authors declare that there are no conflicts of interest 


\section{Tables}

Table 1: Mass range, yield and neurotoxicity of high molecular mass venom fractions

\begin{tabular}{lcccc}
\hline Venom & $\begin{array}{c}\text { Approx molecular } \\
\text { mass range } \\
(\mathrm{kDa})\end{array}$ & $\begin{array}{c}\text { Yield of high mol. } \\
\text { wt. fraction } \\
(\% \mathrm{w} / \mathrm{w})\end{array}$ & $\begin{array}{c}t_{90} \\
(\mathrm{~min})^{*}\end{array}$ & $n$ \\
\hline A. antarcticus (NSW) & $24->66$ & 6.5 & $77 \pm 3$ & 4 \\
A. antarcticus (QLD) & $34->66$ & 12.6 & $63 \pm 3$ & 3 \\
A. antarcticus (WA) & $32->66$ & 6.3 & $59 \pm 5$ & 3 \\
A. antarcticus (SA) & $24->66$ & 2.7 & $>240$ & 3 \\
A. praelongus & $37->66$ & 5.2 & $70 \pm 2$ & 3 \\
A. rugosus & $22-52$ & 5.6 & $85 \pm 3$ & 3 \\
A. laevis & $22-52$ & 14.9 & $73 \pm 3$ & 3 \\
A. wellsi & Not found & - & - & 2 \\
A. pyrrhus & Not found & - & - & 2 \\
\hline
\end{tabular}

${ }^{*} t_{90}$ values represent the mean \pm SEM 
Figures

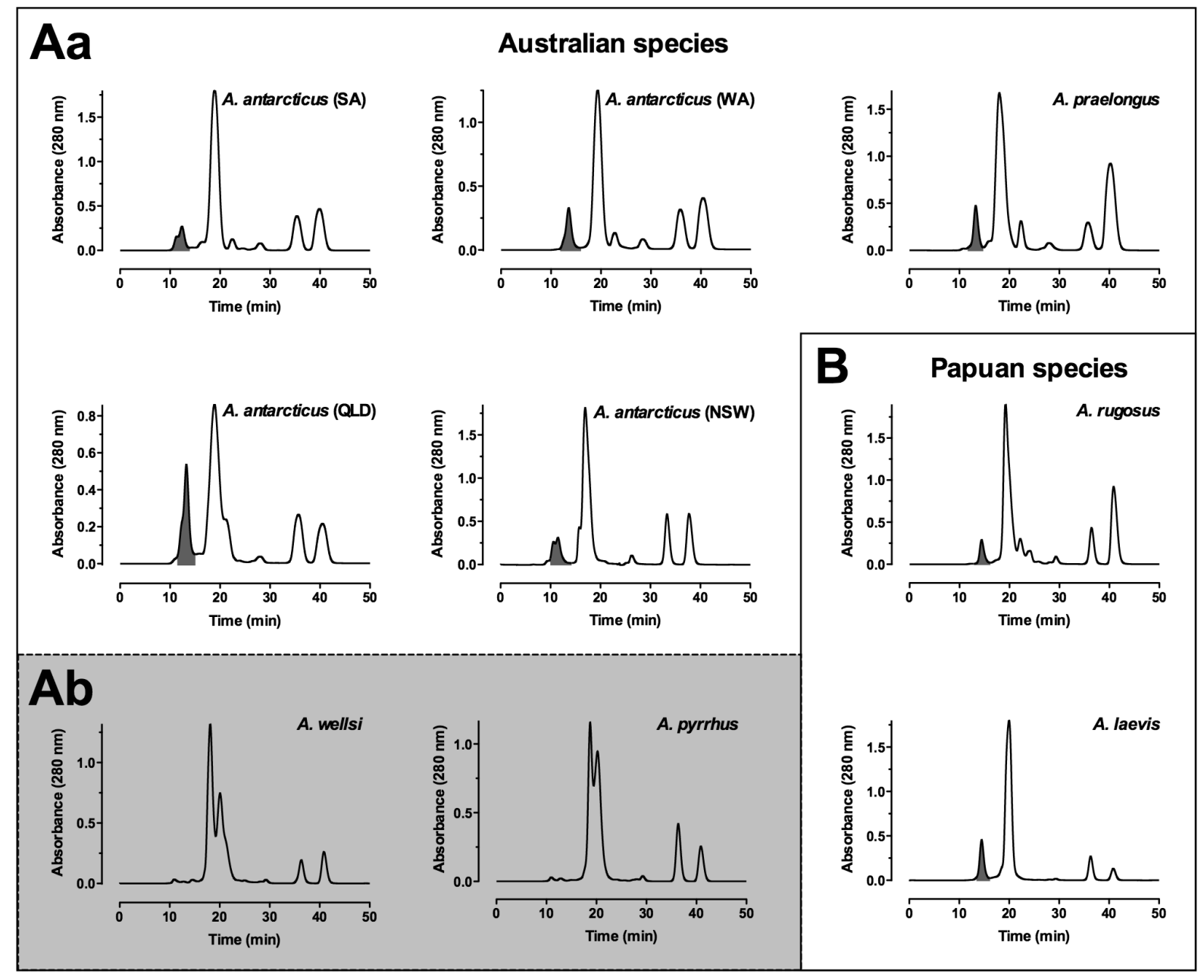

Fig. 1: Representative size-exclusion FPLC chromatograms from Australian (A) and Papuan (B) Acanthophis spp. venoms employing a Superdex G-75 column with an isocratic flow of $0.1 \mathrm{M}$ ammonium acetate $(\mathrm{pH} 6.8)$ at $0.75 \mathrm{ml} / \mathrm{min}$. Shaded fractions on each chromatogram, corresponding to high molecular mass fractions (22 to $>66 \mathrm{kDa}$ ), were collected for further analysis. (Ab) The shaded panel shows chromatograms of $A$. wellsi and $A$. pyrrhus venoms that lack high molecular mass fractions. 


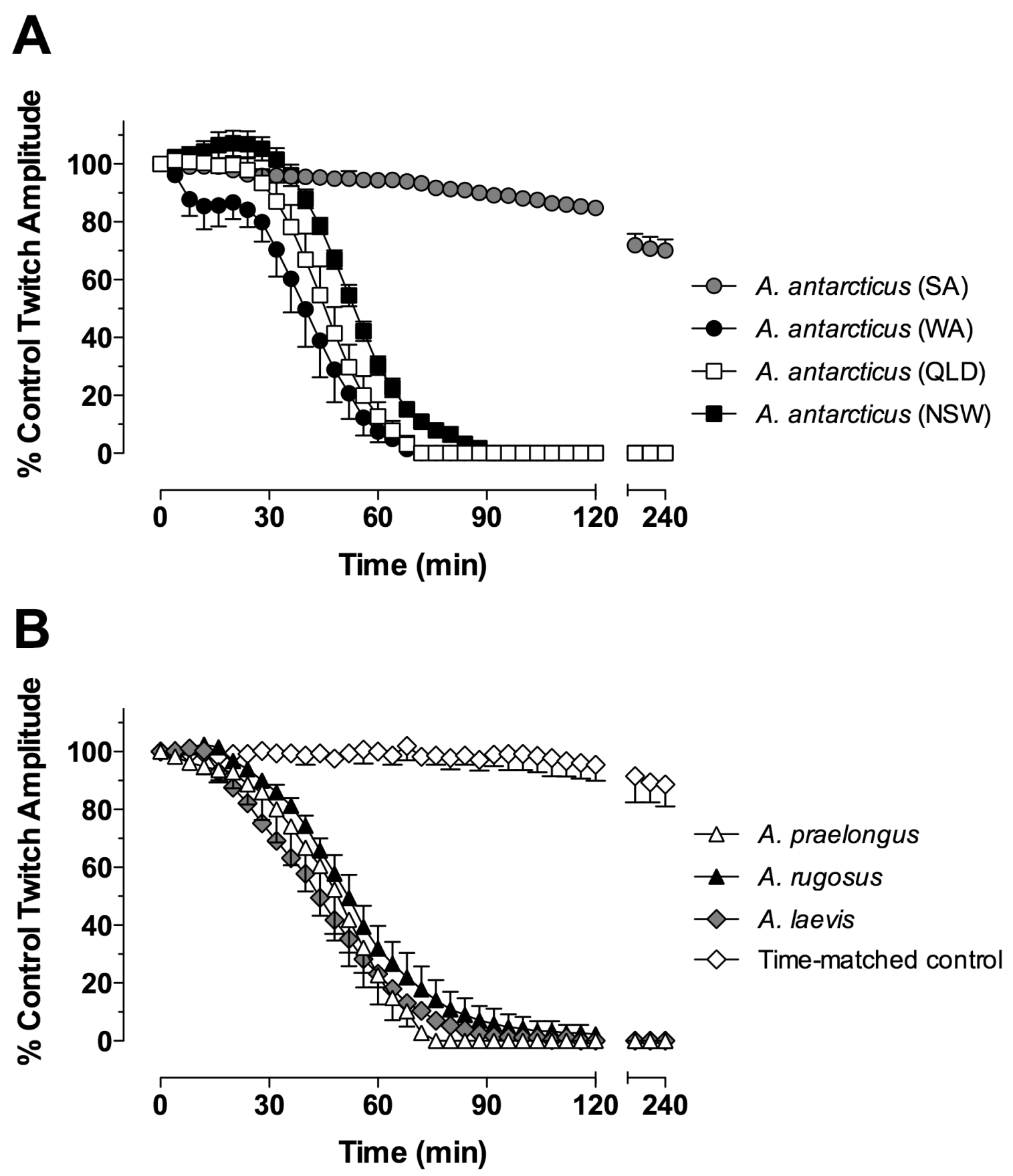

Fig. 2: Inhibition of indirectly-stimulated fast twitch contractions by high molecular mass fractions from Acanthophis spp. venoms in the isolated chick biventer cervicis nervemuscle preparation. (A) Geographic variants of $A$. antarcticus. (B) Remaining Acanthophis species and time-matched (negative) control. All fractions were tested at 
$10 \mu \mathrm{g} / \mathrm{ml}$ and data represents the mean \pm SEM of 4 experiments for $A$. antarcticus (NSW) or 3 experiments for all other venom fractions and negative control.

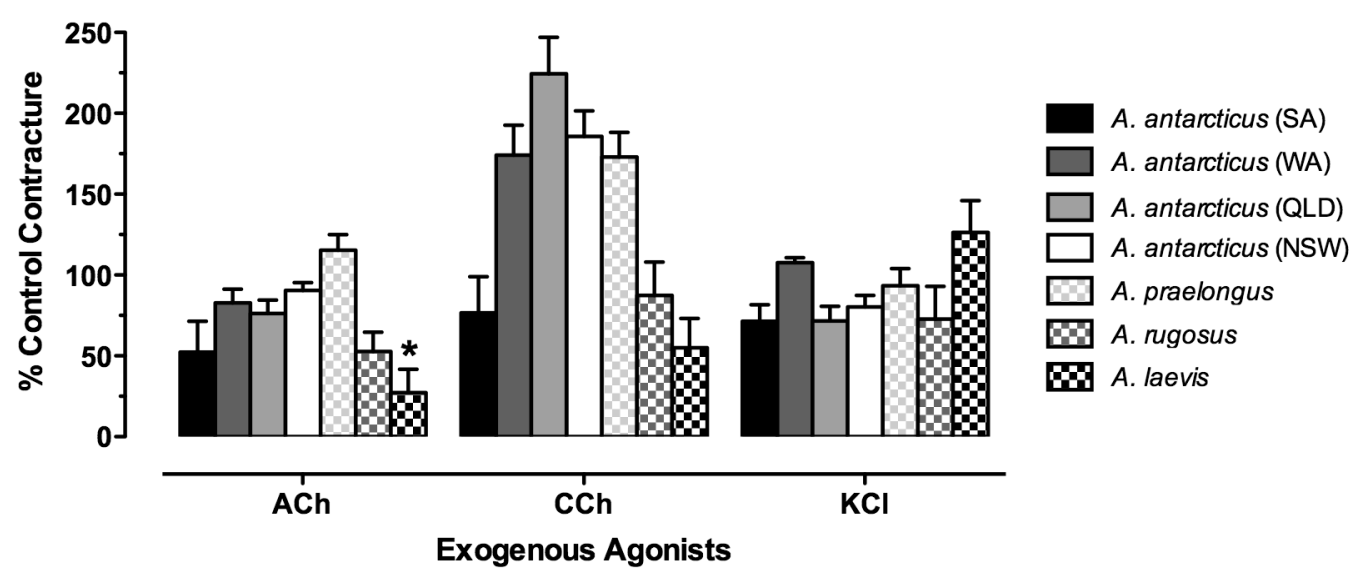

Fig. 3: Effect of high molecular mass fractions from Acanthophis spp. venoms on contractile responses to exogenous $\mathrm{ACh}, \mathrm{CCh}$ and $\mathrm{KCl}$ in the isolated chick biventer cervicis nerve-muscle preparation. Data shows the percentage of control slow fibre contracture responses to exogenous agonists after complete inhibition of nerve-evoked twitch contractions by all high molecular mass fractions. In the case of $A$. antarcticus (SA), data were recorded at $280 \mathrm{~min}$. All fractions were tested at $10 \mu \mathrm{g} / \mathrm{ml}$ and data represents the mean \pm SEM of 4 experiments for $A$. antarcticus (NSW) or 3 experiments for all other venom fractions. ${ }^{*} p<0.01$, significantly different from control response, one-way ANOVA. 


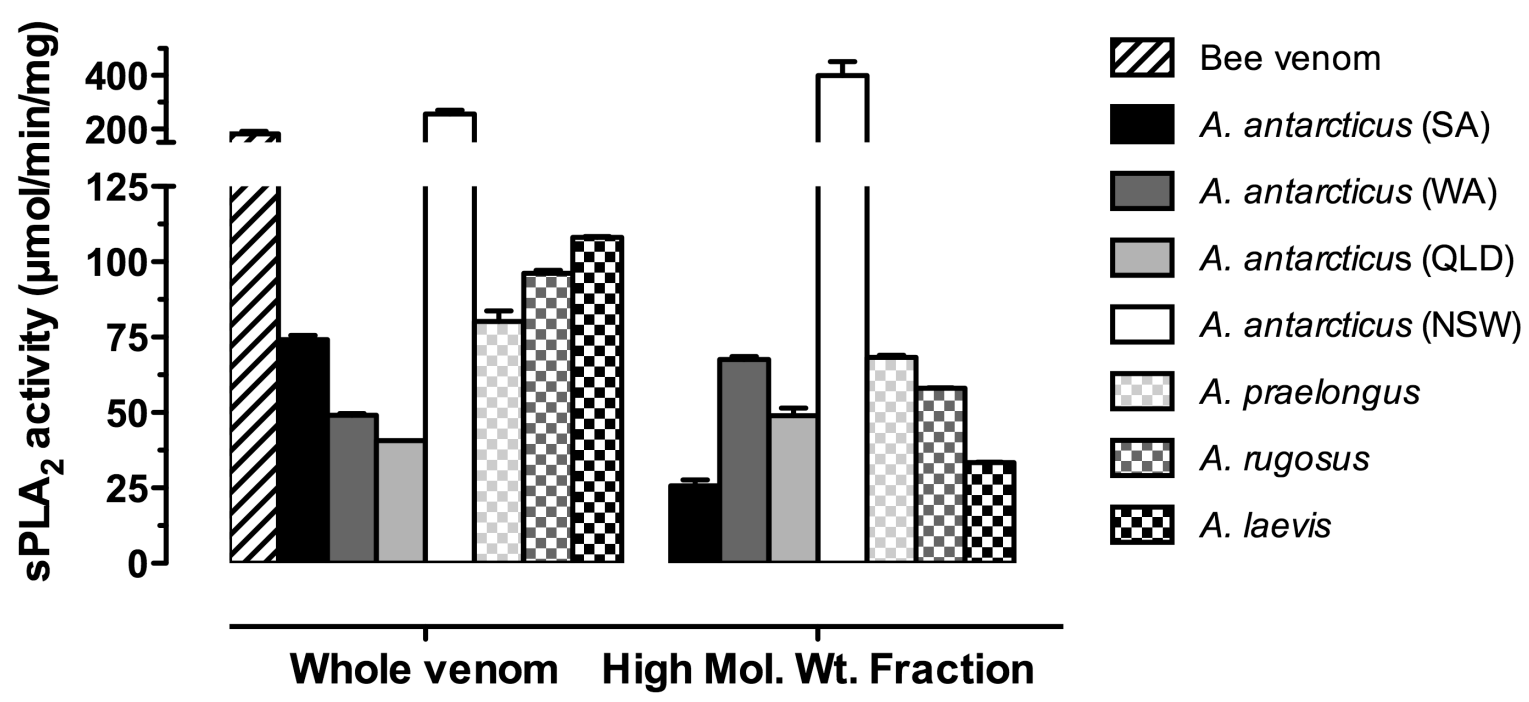

Fig. 4: $\mathrm{PLA}_{2}$ activity of whole Acanthophis spp. venoms (left-hand columns) and their high molecular mass fraction (right-hand columns) as determined using the $\mathrm{sPLA}_{2}$ assay kit. Whole venoms from $A$. wellsi and $A$. pyrrhus were omitted as they did not contain an early eluting peak for toxicity testing. Bee (Apis mellifera) venom was used as a positive control. 

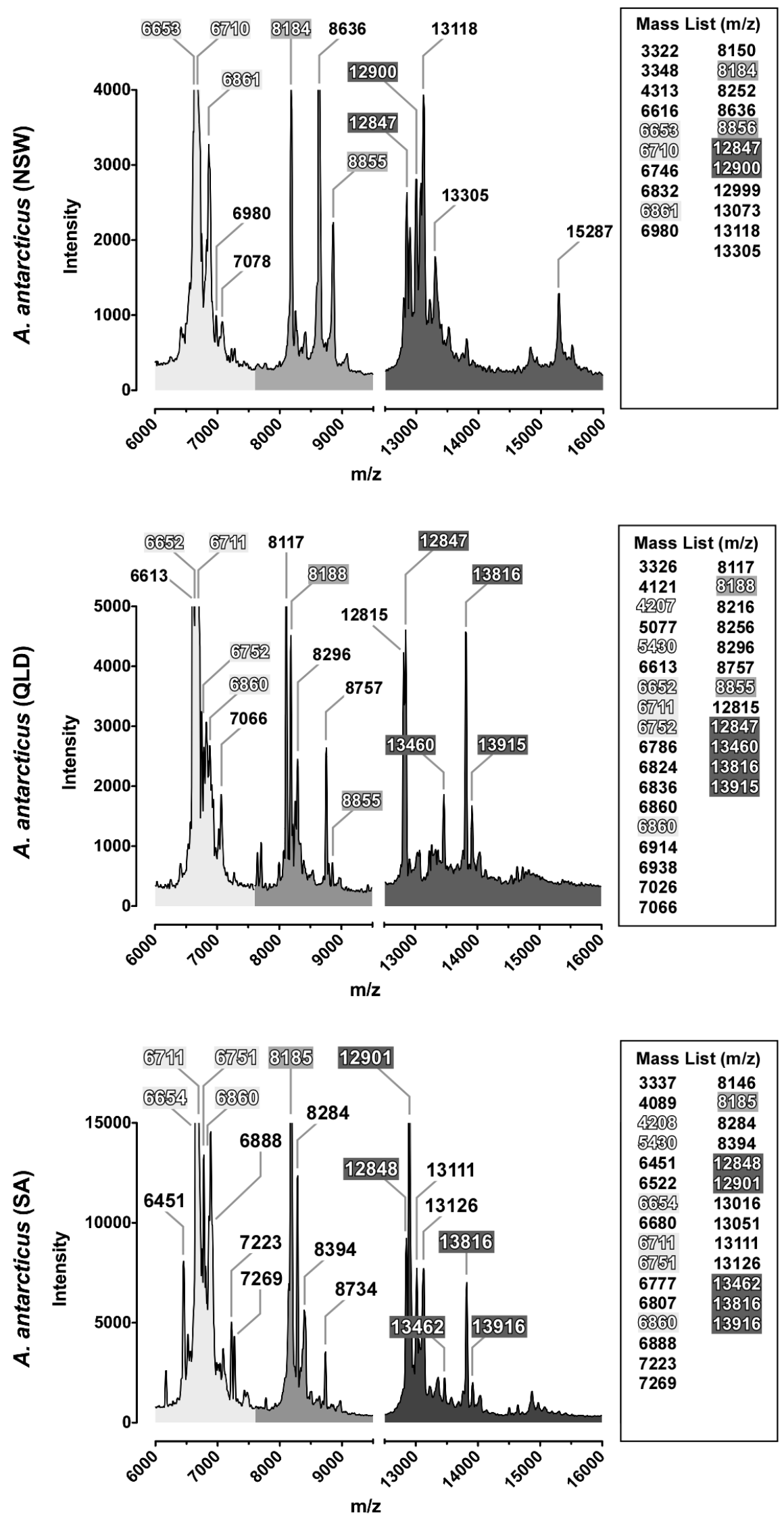
Fig. 5: MALDI-TOF mass spectra of venom from $A$. antarcticus geographic variants. Spectra are focused on masses $(\mathrm{m} / \mathrm{z})$ consistent with the presence of short-chain $\alpha$ neurotoxins ('Short', light grey), long-chain a-neurotoxins ('Long', dark grey) and monomeric PLA 2 neurotoxins ('PLA ${ }_{2}$ ', black). Major peaks are labeled while a complete mass list is shown to the right of each panel. Masses common to at least two venoms are highlighted. 